\title{
Laser application for optical glass polishing
}

\author{
Fidel Vega \\ Núria Lupón \\ Jesús Armengol Cebrian \\ Ferran Laguarta \\ Universitat Politècnica de Catalunya \\ Departament d' Optica I Optometria \\ C/Violinista Vellsolà 37 \\ 08222 Terrassa, Spain \\ E-mail: fvega@oo.upc.es
}

\begin{abstract}
Rapid generation of large area polished optical surfaces by high-power $\mathrm{CO}_{2}$ laser irradiation is shown. Results focus on glasses with high expansion coefficients $\left(\alpha \geqslant 10^{-5} /{ }^{\circ} \mathrm{C}\right)$ conventionally used in the optical industry. The technique involves active beam integration to obtain an intensity irradiation profile with a good uniformity over large spot sizes and is applied to preheated glass samples with initial rms roughness up to $500 \mathrm{~nm}$. To find out the conditions for successful and reliable use of the proposed laser polishing method, the laser-driven heating process was monitored by means of the surface and depth temperature distributions. Whereas the former was determined in situ from the IR radiation emitted by the glass surface, the latter was obtained by comparison of the $\mathrm{IR}$ radiation emitted by the bulk sample with a theoretical model. Laser polishing of $5000 \mathrm{~mm}^{2}$ glass surfaces is reported and processes involved in the modification of the surface texture of the irradiated samples are described and discussed. (C) 1998 Society of Photo-Optical Instrumentation Engineers. [S0091-3286(98)04101-4]
\end{abstract}

Subject terms: high power $\mathrm{CO}_{2}$ laser application; rapid generation of polished glass surface; in-process temperature measurements; laser beam integration.

Paper 34047 received Apr. 30, 1997; revised manuscript received Aug. 12, 1997; accepted for publication Aug. 12, 1997. This paper is a revision of a paper presented at the SPIE conference on Specification, Production, and Testing of Optical Components and Systems, May 1996, Glasgow, Scotland, UK. The paper presented there appears (unrefereed) in SPIE Proceedings Vol. 2775.

\section{Introduction}

The processing of materials based on the melting or softening of a thin surface layer and its later solidification is a topic of longstanding interest. ${ }^{1-3}$ This technique is used to enhance or modify the surface properties and, in particular, to polish rough surfaces. ${ }^{4,5}$ The polishing or, equivalently, the decrease in surface roughness occurs because of a surface tension driven flow in the molten or softened layer. This layer must be at least as deep as the peak-to-valley initial surface roughness. Moreover, the polishing flow occurs only if the surface layer is kept molten or softened for long enough.

Laser polishing of glass is an extraordinarily attractive possibility from an industrial point of view ${ }^{6,7}$ since it would improve the production efficiency of standard components besides allowing the automation of the polishing process. Moreover, it requires no mechanical abrasives or surfacedadapted polishing tools. This technique may be especially suitable for obtaining non-spherical and non-revolution optical polished surfaces, which are very difficult and sometimes even impossible to polish using traditional techniques.

Commonly, laser surface treatments require both the contribution of a large amount of energy on the surface and a controlled and customized beam intensity distribution at the sample plane. These requirements have expanded the use of unstable cavity laser sources that provide high-power nearly pure transverse monomode beams with large spatial coherence. On the other hand, both metallic waveguides ${ }^{8}$ and multifaceted laser-beam integrating mirrors ${ }^{9,10}$ are widely used to obtain flat-top intensity profiles in high- power applications. However, integration of coherent beams results in an intensity profile that shows a sharp contrast because of the interferential effects produced by the superposition of the different portions of the segmented wavefront. In laser applications involving materials with a low thermal conductivity such as glass, the abovementioned effect translates into a non-uniform temperature distribution with high transverse thermal gradients, which would last long enough to induce irregular thermal treatment and/or undesirable effects such as permanent macroscopic surface deformations. ${ }^{11}$

This paper deals with polishing relatively large surfaces of optical glass by high-power $\mathrm{CO}_{2}$ laser irradiation $(\lambda$ $=10.6 \mu \mathrm{m})$. Glass has a very high absorption coefficient at infrared wavelengths longer than $5 \mu \mathrm{m}$, its measured value being in the $10^{5} \mathrm{~m}^{-1}$ range. Moreover, its reflectivity at $10.6 \mu \mathrm{m}$ is around $20 \%$ and therefore the global energy efficiency of $\mathrm{CO}_{2}$ laser irradiation is much higher for glass than for metals. So, when the 10.6- $\mu \mathrm{m}$ radiation emitted by a $\mathrm{CO}_{2}$ laser impinges on a glass surface, a large amount of energy is absorbed in a thin surface layer and the laser treatment can be restricted to the glass surface. Since the bulk sample remains unaffected, it would be possible to polish with no changes in given curvature parameters.

In an earlier work, ${ }^{7}$ the authors studied the laser polishing process of ground ophthalmic lenses with given curvature parameters and surface rms roughness in the $500 \mathrm{~nm}$ range. Their surfaces were irradiated with an $80-\mathrm{mm}^{2} \mathrm{CO}_{2}$ laser spot with a uniform transverse energy distribution, obtained from a $\mathrm{TEM}_{00}$ laser beam expanded and reshaped by means of a vibrating multi-faceted mirror. All processed 
Table 1 Evolution of the TRC-33 glass viscosity versus the temperature.

\begin{tabular}{cc}
\hline \hline $\begin{array}{c}\text { Viscosity } \\
(\mathrm{Pa} \cdot \mathrm{s})\end{array}$ & $\begin{array}{c}\text { Temperature } \\
\left({ }^{\circ} \mathrm{C}\right)\end{array}$ \\
\hline $10^{15.5}$ & 495 \\
$10^{14}$ & 530 \\
$10^{8.65}$ & 705 \\
$10^{6}$ & 885 \\
$10^{5}$ & 995 \\
$10^{4}$ & 1155 \\
\hline \hline
\end{tabular}

lenses were previously heated to a temperature higher than that of the transformation point to avoid irresistible laserinduced thermal stresses. The area of the lens surface under the laser spot appeared polished after the treatment, with rms roughness of approximately $1 \mathrm{~nm}$, and without changes in the initial surface figure. These results support the possibility of polishing larger optical surfaces.

The aim of this paper is the development of a scaled-up application of the polishing method summarized above, always focusing on optical glass areas as large as, for example, a lens surface. To achieve this goal we used a highpower $\mathrm{CO}_{2}$ laser beam that was reshaped by means of a novel active integration method and scanned over the desired area. The laser-driven heating process was real-time controlled by means of two homemade electro-optical sensors. They measured the IR radiation emitted by the surface and the bulk glass samples. From the former, the surface temperature could immediately be determined. From the latter, the depth temperature distribution could be deduced by comparison with a theoretical model. Results show that roughness up to $500 \mathrm{~nm}$ is very efficiently lowered, with final rms values around $1 \mathrm{~nm}$. On the other hand, the waviness that is already presented in the glass surface because of the grinding process can hardly be reduced. Finally it is shown that under certain irradiation conditions a rippling surface deformation of high amplitude can be induced by the scanning of the laser beam over the glass sample, this process being more probably related to the development of high transverse thermal gradients.

\section{Experiments}

\subsection{Large Area Polishing of Optical Glass Surfaces}

The samples treated were circular pieces (diameter $=80 \mathrm{~mm}$ ) of white crown optical glass TRC- $33^{12}$ with concave and spherical surfaces of curvature radius ranging from 60 to $255 \mathrm{~mm}$. Samples with different initial rms surface roughness were studied, the maximum roughness being in the $500 \mathrm{~nm}$ range.

Since glass does not exhibit a well-defined fusion-phase transition, a gradual increase in its temperature induces a progressive decrease in its viscosity and a consequent increase in its ability to flow. The variation of the TRC-33 viscosity with the temperature is given in Table $1 .{ }^{13}$ In accordance with these data, a depth-decreasing temperature distribution induced by a laser surface irradiation in a glass sample translates into a depth-decreasing ability to flow. To

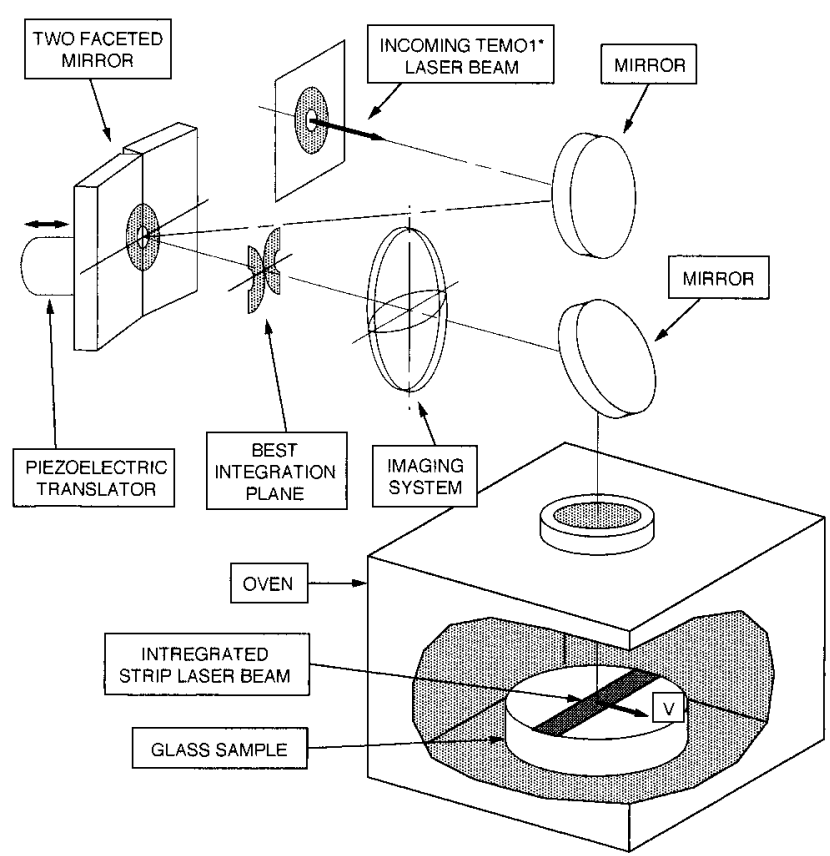

Fig. 1 Schematic drawing of the experimental setup used for laser polishing.

polish, temperature values in the glass surface must typically be above $1000^{\circ} \mathrm{C}$, since only for these values is the glass viscosity coefficient small enough $\left(\$ 10^{4} \mathrm{~Pa} \cdot \mathrm{s}\right)$ for a surface-tension driven mass flow to be established. Moreover, to avoid a global deformation, temperature values in the bulk sample must be kept below the glass softening temperature $\left(\approx 650^{\circ} \mathrm{C}\right)$.

As was mentioned above, to minimize the occurrence of high transverse thermal gradients on the glass surface a uniform laser intensity distribution is needed. Such a distribution is usually obtained by means of a suitable integration system. In the authors' earlier work, ${ }^{7}$ beam integration was obtained by means of a vibrating multi-faceted mirror. The size of the flat facets limited the size of the treated area, which in our case was $80 \mathrm{~mm}^{2}$. To irradiate larger surfaces $\left(>5000 \mathrm{~mm}^{2}\right)$ a complete scaling-up of this experiment might have been carried out to obtain a larger spot at the sample plane. This would have required a higher power laser, a very large aperture beam expander, and very large facets in the integrating mirror. With such constituents the dimension of the experimental setup would have been enormous and its cost very high. Moreover, a suitable vibrating system to integrate the beam could hardly have been found. Therefore, we developed a new active integration technique ${ }^{14}$ that is a basic part of the irradiation setup shown in Fig. 1.

The $\mathrm{CO}_{2}$ laser used delivers a highly spatially-coherent incoming beam with a transverse energy distribution corresponding roughly to the $\mathrm{TEM}_{01}^{*}$ mode (Fig. 2) and with a maximum power of $2 \mathrm{KW}$. The active integration system is based on a mirror with two facets, one of which is able to vibrate under the action of a piezoelectric translator. The faceted mirror divides the incoming beam into two beamlets that are forced to overlap partially in order to obtain a redistribution of the beam intensity for greater uniformity. 


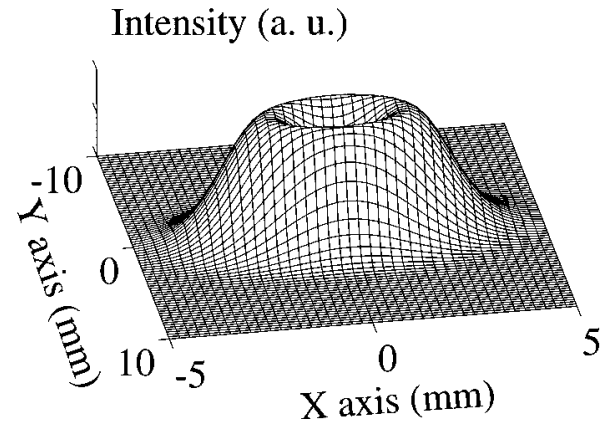

Fig. 2 Simulated intensity distribution of the incoming $\mathrm{TEM}_{01}^{*}$ laser mode beam.

This distribution is shown in Fig. 3(a) and leads to a fairly uniform profile when integration in the $Y$ direction is carried out [Fig. 3(b)]. It is worth noting that this is equivalent to sweeping the intensity distribution over the sample as shown in Fig. 1. However, the interferential effects produced by the superposition of the two coherent beamlets must result in large intensity variation. A simulation of the integrated profile under these conditions is shown in Fig. 4. Calculations were done by taking into account the optical path difference between the reflected beamlets. This is similar to the coherent addition of the wavefronts reflected in a Fresnel's mirror. ${ }^{15}$ Visibility of the fringes varies along the $X$ axis, the maximum contrast occurring at the center of the profile, where the intensity of the overlapping beamlets is similar. By activating the piezoelectric translator, one of the facets moves periodically and a periodic phase shift between the two reflected beamlets is established. In con-
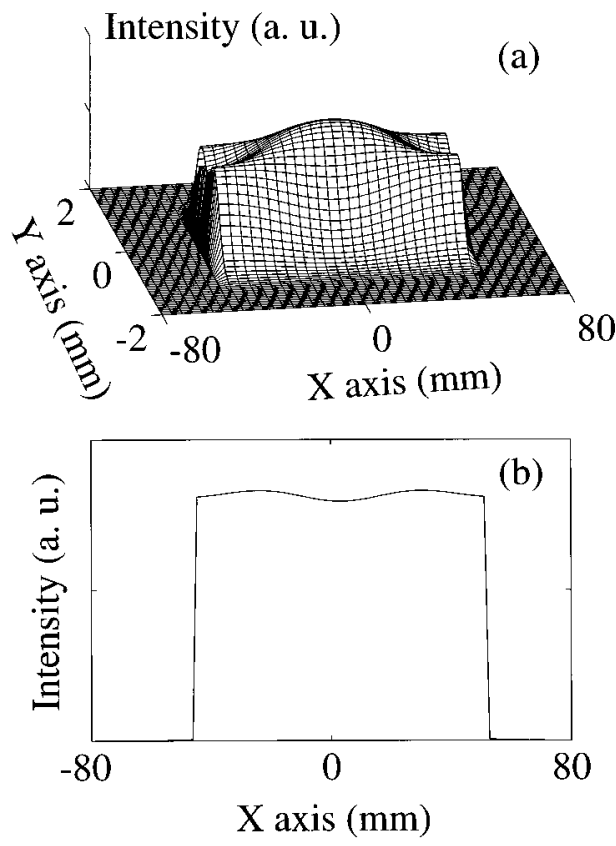

Fig. 3 Simulated intensity distribution of the strip laser beam obtained at the sample plane as the two reflected beamlets spatially overlap (a), and intensity profile after integration of the intensity distribution in the $Y$ direction (b). Interferential effects are not included in the calculations.

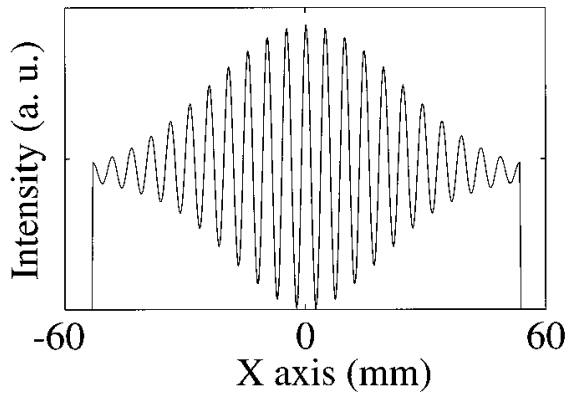

Fig. 4 Simulated intensity profile of the strip laser beam obtained at the sample plane including interferential effects between the two reflected beamlets.

sequence the interference pattern vibrates at the same frequency as the facet and its contribution to the intensity profile averages out. The experimental intensity profiles obtained are shown in Fig. 5. They were recorded by placing a 1-mm-wide slit in front of a pyroelectric detector. It is worth noting that the measured profile without piezotranslator [Fig. 5(a)] presents a fringes visibility lower than expected from calculations (see Fig. 4). This may be due to both the finite width of the used slit, and the deviations in the laser mode of the $\mathrm{TEM}_{01}^{*}$ one. Upon active integration [Fig. 5(b)], there is a strong reduction in the visibility of the interference fringes of up to $5 \%$. Finally, the combination of the faceted mirror and an optical imaging system results in an intensity profile with good uniformity over a large strip beam. The imaging system is formed by two $\mathrm{ZnSe}$ lenses with antireflecting coatings for $\lambda=10.6 \mu \mathrm{m}$. The first lens is a converging cylindrical one $(f=25 \mathrm{~cm})$ whereas the second is a diverging spherical lens $(f=-12.5 \mathrm{~cm})$. By changing the distance between the

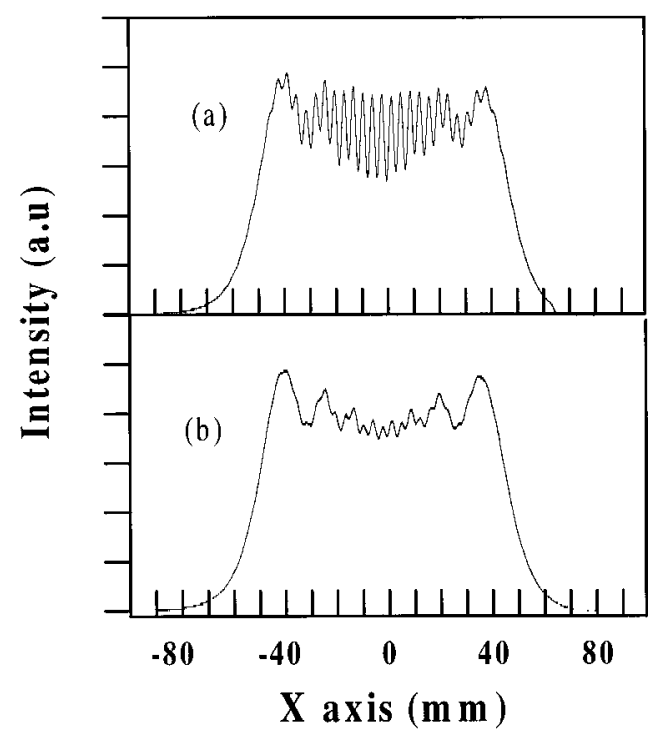

Fig. 5 Experimental intensity profiles obtained along the largest dimension of the strip laser beam at the sample plane without (a), and (b) under active integration. Profiles were recorded by using a 1.0$\mathrm{mm}$-wide slit positioned in front of a pyroelectric detector and scanning the laser beam along its larger dimension. 


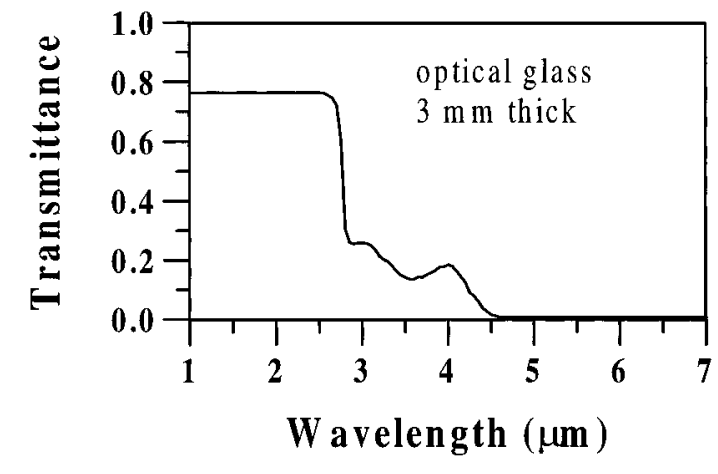

Fig. 6 Measured spectral transmittance of optical glass.

lenses the beam dimension at the sample plane can vary from $2 \times 100 \mathrm{~mm}^{2}$ to $18 \times 100 \mathrm{~mm}^{2}$.

Due to laser treatment, depth thermal gradients always appear in glass because of the great absorption and the low thermal diffusivity mentioned above. As the thermal expansion coefficient depends on temperature, these high depth thermal gradients promote internal stresses in a treated glass sample and might make it crack. ${ }^{6}$ These undesirable effects worsen when the temperature is around the transformation point $\left(T_{g}=530^{\circ} \mathrm{C}\right)$, since the thermal expansion coefficient increases suddenly at this point. Therefore, samples must be preheated to a temperature slightly higher than $T_{g}$. Also, after the laser treatment, during cooling, the temperature of the sample must go through the transformation point in a controlled way, following a typical annealing cycle. To satisfy these needs, the laser treatment is carried out in an oven as shown in Fig. 1.

\subsection{Control of the Laser-Driven Heating Process}

To obtain on-line control of the heating cycle of a glass sample during the laser polishing process, two homemade electro-optical sensors are used to determine respectively the surface and depth temperature distribution from the IR radiation emitted by the glass sample while it is being irradiated.

Figure 6 shows the measured glass spectral transmittance. It shows that glass behaves as a semi- transparent material for infrared wavelengths $\lambda<5 \mu \mathrm{m}$ and as an almost perfect absorbent for $5 \mu \mathrm{m} \leqslant \lambda$. Therefore, radiation emitted by a heated glass sample with $5 \mu \mathrm{m} \leqslant \lambda$ arises exclusively from a thin surface layer, whereas radiation of wavelengths $\lambda<5 \mu \mathrm{m}$ arises also from the bulk of the glass. Since the infrared emission depends only on the temperature of the emitter, to find out the surface-temperature distribution a sensor sensitive to $\lambda \geqslant 5 \mu \mathrm{m}$ radiation (sensor $A$ ) was developed, and to find out the depth-temperature distribution a second one (sensor $B$ ) sensitive to $\lambda<5 \mu \mathrm{m}$ was developed. To monitor the laser-driven heating process, with sensor $A$ or $B$, we used the experimental setup shown in Fig. 7.

The fundamental constituent of sensor $A$ is a pyroelectric detector with a bandpass interferential filter between 5 and $6.5 \mu \mathrm{m}$. The optical system of the sensor is an uncoated $\mathrm{ZnSe}$ meniscus lens with a $62.5-\mathrm{mm}$ focal length. A reduced aperture allows this system to image, without no-

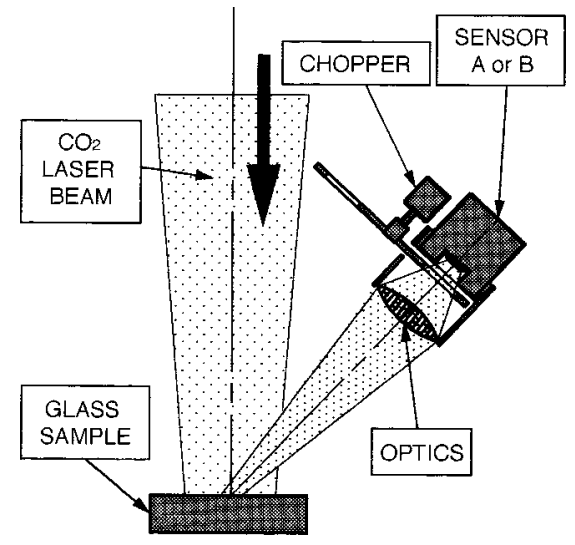

Fig. 7 Schematic drawing of the experimental setup used to monitor the sample temperature.

ticeable aberrations, the surface of the sample on that of the detector, with a 2-mm spatial resolution and maintaining an acceptable signal-to-noise ratio. The lens and the detector are mounted on the two ends of a cylindrical header, which is the mechanical support of the sensor. The incoming radiation to the detector is mechanically chopped and the detector signal is electronically treated by a lock-in amplifier. The response time of the sensor is $50 \mathrm{~ms}$.

The response of sensor $A$ depending on temperature has been calibrated by means of the radiation emitted by a black body and considering the attributes of the sensor, namely, the aperture, the lens and bandpass filter transmittance, the overall geometry and the spectral responsivity.

The setup and operation of sensor $B$ are the same as those of $A$. Its fundamental constituent is a $\mathrm{PbSe}$ photoconductive detector with a detection range from 1.5 to $4.8 \mu \mathrm{m}$. This detector was selected because in its detection range the sensitivity to temperature changes between 500 and $1000^{\circ} \mathrm{C}$ is the highest, and the in-depth temperature of glass during laser polishing varies typically between these two values. The optics of the sensor is an IRGN-6 lens with a $20-\mathrm{mm}$ focal length that is nearly transparent to the wavelengths to which the detector is sensitive.

The signal in sensor $B$ is indirectly related to the depth temperature distribution and its temporal evolution in the sample treated. The laser-driven thermal process in the sample is simulated by a theoretical model based on the heat-conduction transfer equation. The sample temperature is calculated as a function of depth and time, starting from the laser power applied, its temporal evolution and the thermal and optical parameters of the glass. The computed temperatures of the sample, the thermal radiation laws, and the attributes of the sensor allow us to calculate the theoretical sensor signal. Further details on the sensor features and thermal simulations can be found elsewhere. ${ }^{7,11}$

Figure 8 shows the recorded signal corresponding to a fixed point of the surface during a laser polishing experiment. In this measure, the sensor always imaged the same point of the surface while the laser scans the sample. Results indicate an initial surface temperature of roughly $580^{\circ} \mathrm{C}$ (pre-heating temperature) and a maximum of $1250^{\circ} \mathrm{C}$ upon laser irradiation. The cooling tail from maximum to initial temperature lasted approximately $35 \mathrm{~s}$, the 


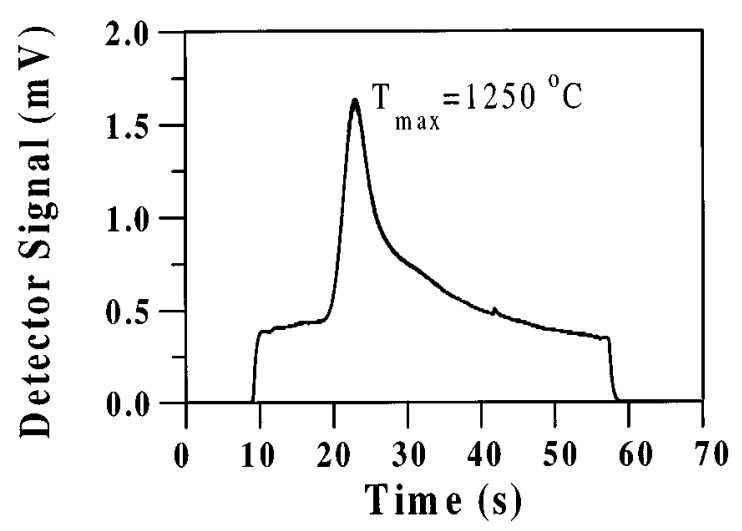

Fig. 8 Temporal evolution of the temperature of a point on the glass surface during a laser polishing experiment. The laser strip beam was focused to a width of $2 \mathrm{~mm}$, the laser power density was $500 \mathrm{~W} / \mathrm{cm}^{2}$ and the irradiation time of a surface point was $1.33 \mathrm{~s}$.

highest decrease occurring during the first two seconds of the cooling process.

Figure 9 shows the recorded signal corresponding to a surface temperature measurement carried out along the glass surface without [Fig. 9(a)] and with [Fig. 9(b)] laser irradiation. In this experiment the sensor scans the sample surface, thus imaging each time a different point of the sample surface. From Fig. 9(a) one can conclude that the sample is fairly uniformly pre-heated in the oven. Upon laser irradiation, the incidence point of the laser beam strip is imaged throughout polishing by making equal both the sensor and laser scan velocities. Results show that the temperature of the irradiated point is nearly constant all over the sample surface [Fig. 9(b)]. Since this result was proved to be independent of the position of the imaged point along the larger dimension of the strip beam, one can conclude that there is a good thermal uniformity over the entire surface treated.

\section{Results and Discussion}

In accordance with Ref. 16 the surface texture of the samples (i.e., the repetitive and/or random deviations from

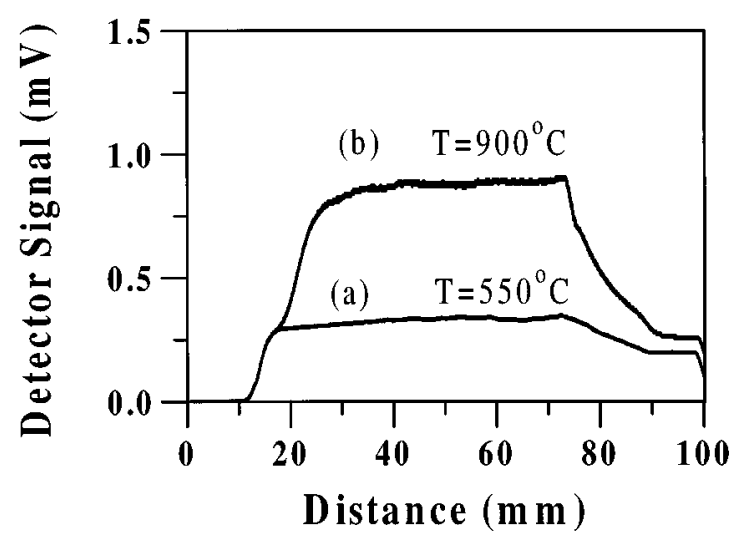

Fig. 9 Temperature along the glass sample surface (a), and maximum temperature achieved along the glass sample surface under laser irradiation (b). The laser strip beam was focused to a width of $2 \mathrm{~mm}$, the laser power density was $500 \mathrm{~W} / \mathrm{cm}^{2}$ and the irradiation time of a surface point was $0.4 \mathrm{~s}$.

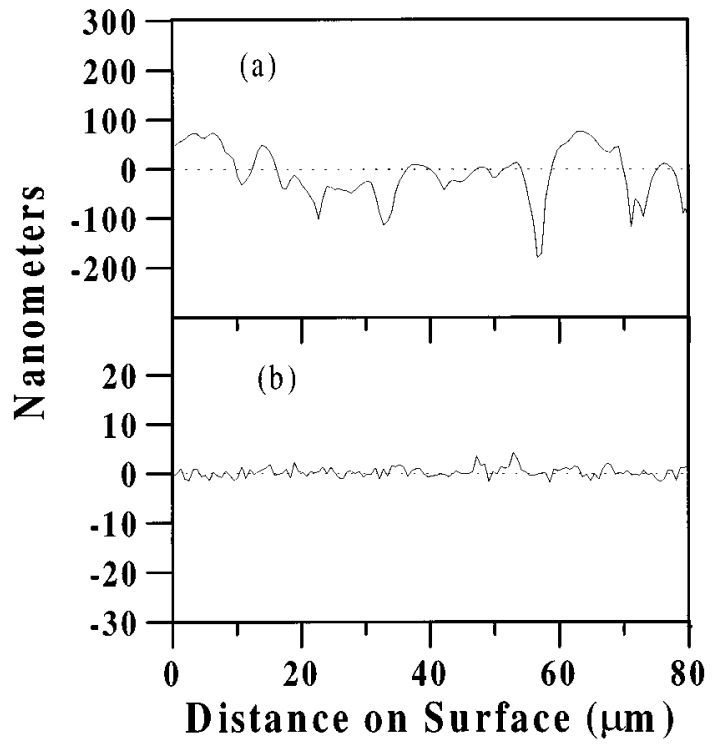

Fig. 10 Roughness profiles obtained with a $40 X$ Mirau objective in a ground glass surface (a) and after laser irradiation (b). Notice the differences between the vertical scales. The laser strip beam was focused to a width of $18 \mathrm{~mm}$, the laser power density was $94.4 \mathrm{~W} / \mathrm{cm}^{2}$ and the irradiation time of a surface point was $9 \mathrm{~s}$.

the nominal surface that forms the three-dimensional topography of the surface) is analyzed in terms of roughness and waviness. Roughness is formed by the finer irregularities of high spatial frequency of the surface, whereas waviness refers to the more widely spaced components of the surface texture. Since roughness is considered to be superimposed on the waviness profile, each kind of defect has to be measured in its own sampling length. To do that we use a phase-shifting optical profiler with different objectives: a $40 X$ magnification Mirau for measuring the sample roughness with nanometer resolution, and a $2.5 \mathrm{X}$ objective featuring a Michelson interferometer to analyze the waviness. Since the measured surface profile includes the surface texture that is superimposed on this profile, to show the surface texture, a nominal profile (exclusive of any intended surface texture) is subtracted from the measured one. Then, roughness and waviness are displayed as deviations from the nominal profile.

Figures 10(a) and 10(b) show, respectively, the roughness of a ground sample before and after laser treatment. The plot in Fig. 10(a) shows an average distance between peaks (or valleys) of around 17 to $18 \mu \mathrm{m}$, with a peak-tovalley height in the 260-nm range. This topography is caused by the grinding of the surface of the samples. Figure 10(b) shows that after laser treatment the sample roughness has decreased up to rms values of $1 \mathrm{~nm}$ with a maximum peak to valley height of $6 \mathrm{~nm}$. It must be emphasized that this dramatic reduction in the reduction in the roughness was achieved all over the treated area of the glass sample $\left(\approx 5000 \mathrm{~mm}^{2}\right)$.

During the grinding process of the samples, randomly distributed waviness can be generated over the surface leading to noticeable variations in the surface profile with respect to the nominal one. The ability of the laser polishing process to eliminate such defects was also investigated. 


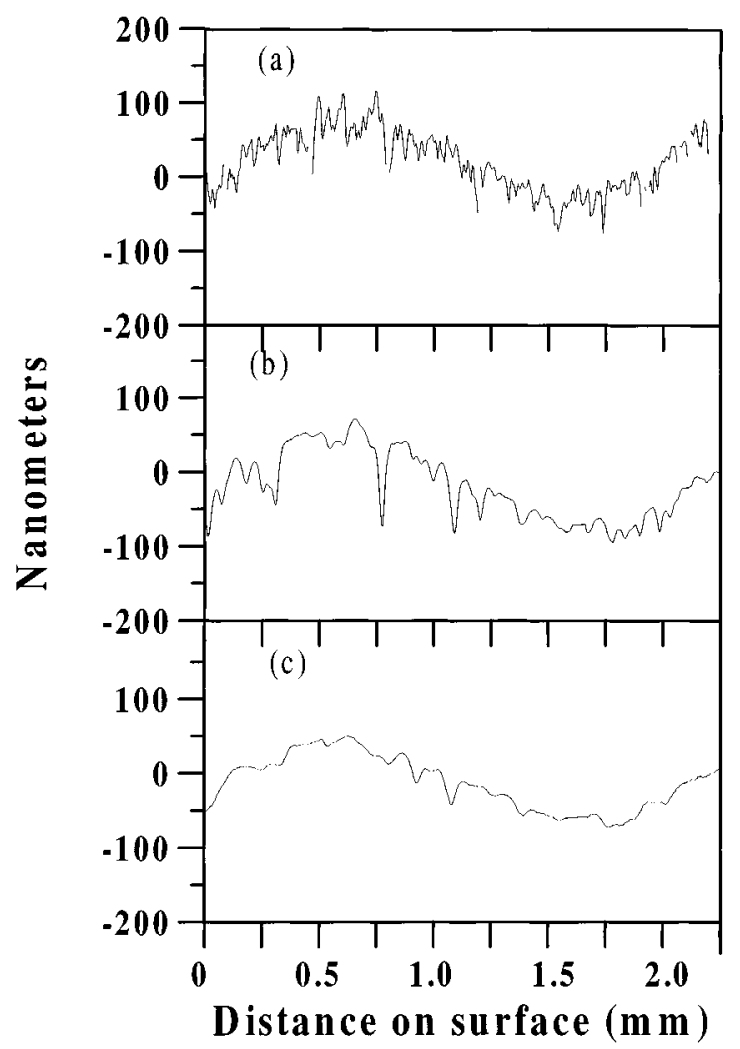

Fig. 11 Waviness profiles obtained with a 2.5X Michelson objective in a ground glass surface (a) and after one (b) and two (c) laser irradiations. The laser strip beam was focused to a width of $18 \mathrm{~mm}$, the laser power density was $83.3 \mathrm{~W} / \mathrm{cm}^{2}$ and the irradiation time of a surface point was $3 \mathrm{~s}$.

Figure 11(a) shows typical waviness that is impressed in a ground sample. A waviness height of $\approx 140 \mathrm{~nm}$ is found with a waviness spacing in the $2-\mathrm{mm}$ range. Notice that the superimposed roughness pattern presents interruptions in the sharpest peaks and valleys because the $2.5 \mathrm{X}$ objective cannot measure defects with a slope greater than $2.5^{\circ}$. This sample was irradiated twice with the widest strip laser beam $(\approx 18 \mathrm{~mm})$ to include several waves in its action. The surface profiles measured after the first and second laser treatments are shown in Figs. 11(b) and 11(c), respectively. Again, a clear reduction in roughness happens with the deepest valleys being progressively filled, but on the other hand, the waviness remains nearly unaffected after the laser treatment, even when the initial waviness height $(\approx 140 \mathrm{~nm})$ was lower than the peak-to-valley roughness $(\approx 260 \mathrm{~nm})$.

The difference between the high efficiency of the laser treatment in eliminating the roughness and its low capability to reduce the waviness can be understood by taking into account that the higher the slope of a defect of the surface, the higher should be the efficiency of the surface-tension driven mass flow. By estimating the slope of the surface as the ratio between defect height and defect spatial length, slope values in the $10^{-2}$ range or higher are typically found for the roughness, whereas the larger spatial extension of the waviness leads to slopes in the $10^{-4}$ range. Then, in the present experimental conditions with a strip laser beam

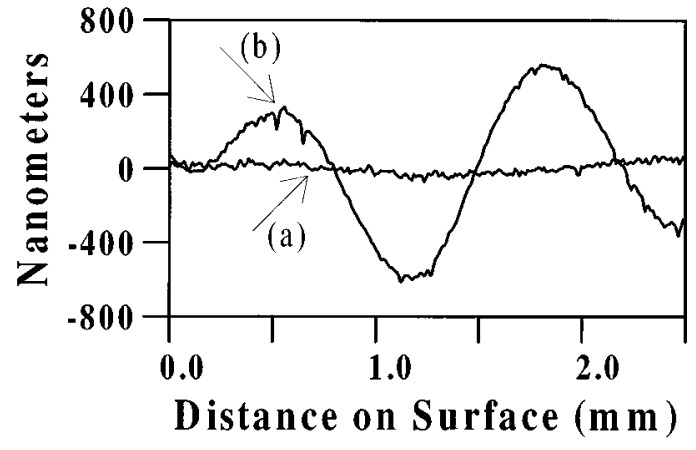

Fig. 12 Ground profile (a) and laser induced ripples (b) obtained by irradiation with a 2-mm-width strip beam, laser power density $100 \mathrm{~W} / \mathrm{cm}^{2}$ and irradiation time of a surface point of $2.5 \mathrm{~s}$. Measurements were obtained with the $2.5 X$ Michelson objective.

scanning the surface of the samples, the action of the surface tension during the irradiation time is small enough to reduce the waviness. Enhancement of the surface tension driven mass flow by increasing the energy deposited on the sample surface induced a macroscopic deformation of the surface figure.

The influence of the irradiation parameters (i.e., laser power, the width of the laser beam and the scanning velocity) on the modification of the surface texture of the glass samples upon irradiation was studied. In particular by using strongly focused strip beams, the laser treatment can induce the formation of ripples of high amplitude. Such a pattern, which has not been observed in the small-scale experiments, always appears perpendicular to the scanning direction and parallel to the strip laser beam. Figure 12 shows both the ground surface profile and the resultant ripples after laser irradiation, the latter showing a peak to valley height around $1.3 \mu \mathrm{m}$ with a ripple wavelength of $\approx 1.5 \mathrm{~mm}$. Rippling effects with periodicity of the order of the laser wavelength have been widely reported during laser irradiation of metals, semiconductors and dielectric surfaces. ${ }^{17-19}$ They are generated because of the interference of the incident and scattered optical waves, the latter arising from defects at the surface. Larger-scale undulations have also been observed upon laser melting and alloying of metals and semiconductors. ${ }^{20,21}$ They are usually explained in terms of surface-tension gradients that result in a surface flow. Such gradients are induced by the transverse thermal gradient between the hotter zone of the beam and the colder periphery. ${ }^{22}$ To check this scenario we carried out several irradiations with different widths of the laser beam strip. The energy deposited on the sample is kept constant by modifying both the laser power and the laser scan velocity. The formation of ripples was induced when the width of the laser beam strip is strongly reduced, a situation for which high transverse thermal gradients are expected in the direction of scanning. Moreover, since it was experimentally stated that even for strongly focused beams the temperature of the points of the surface during the laser treatment is fairly uniform (Fig. 9), we can discard the rippling generation because of non-uniform heating of the surface.

The preceding discussion emphasizes that laser polishing induces a complex three-dimensional dynamics on the samples that depends on the spatially and temporally in- 
duced temperature distribution and the initial surface texture. Our results indicate that to eliminate waviness, the ability of the surface to flow should be enhanced while keeping the surface figure constant. Moreover, minimization of the transverse thermal gradients on the sample surface is of main importance for the success of the application.

\section{Conclusions}

A new $\mathrm{CO}_{2}$ laser application to polish large areas of optical surfaces in conventional glasses used by the optical industry has been proposed and proved. By means of a new laser beam integration method, fairly uniform surface heating over large areas is achieved. The laser-induced thermal cycle has been successfully monitored. The roughness of high spatial frequency of the ground surfaces is quite efficiently lowered because of the action of the surface tension. Starting with samples of rms roughness in the $500 \mathrm{~nm}$ range, roughness around $1 \mathrm{~nm}$ can be obtained by laser treatment. On the other hand, defects such as waviness that present a reduced slope are hardly reduced because of the lowering of the efficiency of the surface tension-driven mass flow. By inducing high transverse thermal gradients, the formation of ripples on the irradiated surfaces is found

\section{Acknowledgments}

Industria de Óptica S. A. (INDO) and Essilor International are acknowledged for financial support. This work was also supported by the Comisión Interministerial de Ciencia y Tecnología (CICYT, Ref. PTR93-0024), Spain. The authors thank A. Savall, J. L. Mercier, and G. Sagot for helpful discussions.

\section{References}

1. E. M. Breinan and B. H. Kear, "Rapid solidification laser processing at high power density,', Laser Materials Processing, M. Bass, Ed., Vol. 3, pp. 235-295, North-Holland (1983).

2. J. Mazumder, "Overview of melt dynamics in laser processing," Opt. Eng. 30(8), 1208-1219 (1991).

3. See: J. M. Pelltier, "Laser surface treatment: state of the art and prospect," High Power Lasers: Applications and Emerging Applications, Proc. SPIE, G. Sayegh and M. R. Osborne, Eds., 2789, 54-64 (1996) and references contained thereby.

4. P. A. Temple, W. H. Lowdermilk, and D. Milam, "Carbon dioxide laser polishing of fused silica surfaces for increased laser-damage resistance at $1064 \mathrm{~nm}, "$ Appl. Opt. 21(18), 3249-3255 (1982).

5. A. Bosseboeuf, J. Boulmer, J. P. Budin, and D. Debarre, "Planarization of rough Si surfaces by laser annealing," Appl. Surf. Sci. 109110, 473-476 (1997).

6. Y. M. Xiao and M. Bass, "Thermal stress limitations to laser fire polishing of glasses," Appl. Opt. 22(18), 2933-2936 (1983).

7. F. Laguarta, N. Lupon, and J. Armengol, "Optical glass polishing by controlled laser surface-heat treatment," Appl. Opt. 33(27), 65086513 (1994)

8. J. M. Geary, "Channel integrator for laser beam uniformity on target,' Opt. Eng. 27(11), 972-977 (1988).

9. D. M. Dagenais, J. A. Woodroffe, and I. Itzkan, "Optical beam shaping of a high power laser for uniform target illumination," Appl. Opt. 24(5), 671-675 (1987)

10. F. M. Dickey and B. D. O'Neil, "Multifaceted laser beam integrators: general formulation and design concepts," Opt. Eng. 27(11), 9991007 (1988)

11. J. L. Ocaña, A. García-Beltrán, F. Laguarta, N. Lupón, J. Armengol, and F. Vega, "Analysis of the effect of optically induced local nonuniformities in laser surface treatment applications," Lasers in Material Processing, CLEO/Europe EQUEC'96, 8-13 September 1996, Hamburg, Germany.

12. Glass commercially available from Corning France S. A. Optical Division, 6 rue du Gâtinais-B. P. n³, 77167 Bagneaux-Sur-Loing, France.
13. Technical Data Handbook, Corning France S. A. Optical Division (October 1994).

14. J. Armengol, F. Vega, N. Lupón, and F. Laguarta. "A two-faceted mirror for active integration of coherent high power laser beams," Appl. Opt. 36(3), 658-661 (1997).

15. E. Hecht and A. Zajac, Optics, Addison-Wesley, Reading, MA (1974).

16. Surface Texture (Surface Roughness, Waviness, and Lay), An American National Standard ANSI/ASME B46.1, published by The American Society of Mechanical Engineers (1985).

17. G. N. Maracas, G. L. Harris, C. A. Lee, and R. A. McFarlane, "On the origin of periodic surface structure of laser-annealed semiconductors,'” App. Phys. Lett. 33(5), 453-455 (1978).

18. Z. Guosheng, P. M. Fauchet, and A. E. Siegman, "Growth of spontaneous periodic surface structures on solids during laser illumination,'” Phys. Rev. B 26(10), 5366-5381 (1982).

19. M. J. Soileau, "Ripple structures associated with ordered surface defects in dielectrics," IEEE J. Quantum Electronics 20(5), 464-467 (1984)

20. T. R. Anthony and H. E. Cline, "Surface rippling induced by surfacetension gradients during laser surface melting and alloying," J. Appl. Phys. 48(9), 3888-3894 (1977).

21. V. N. Tokarev and V. I. Konov, "Suppression of thermocapillary waves in laser melting of metals and semiconductors," J. Appl. Phys. 76(2), 800-805 (1994).

22. J. Calatroni and G. Da Costa, "Interferometric determination of the surface profile of a liquid heated by a laser beam," Opt. Comm. 42(1), 5-9 (1982).

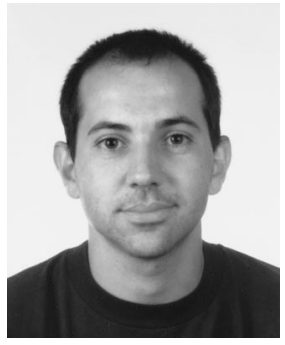

Fidel Vega received his MS in 1988 and $\mathrm{PhD}$ in 1993 from the Universidad Complutense (Madrid). From 1990 to 1993 he was a student fellow at the Laser Material Processing and Optical Storage Group at the Instituto de Optica (C.S.I.C, Madrid). $\mathrm{He}$ is currently professor of optics in the Department of Optics and Optometry at the Universidad Politècnica de Catalunya (Spain). His major research areas are laser material processing (mainly semiconductors and glasses), laser beam-shaping and optical surface profilometry and metrology.

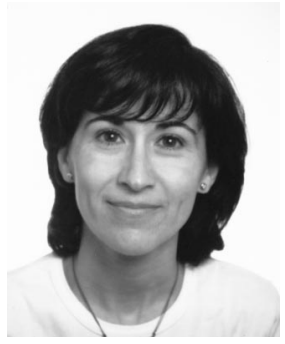

Núria Lupón received her MS in 1980 from the Universitat Autònoma de Barcelona. Since 1984 she has been a professor of physics in the Department of Optics and Optometry at the Universitat Politècnica de Catalunya (Spain). In this department she is also working toward her $\mathrm{PhD}$ degree in the fields of laser material processing and laser beam-shaping.

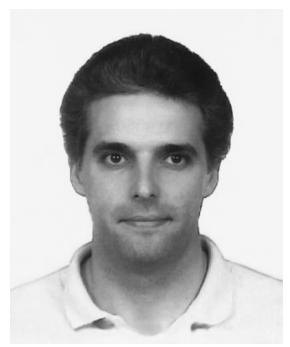

Jesús Armengol Cebrian is professor of optics in the Department of Optics and Optometry at the Universidad Politécnica de Catalunya. He received the MS degree in physics from the Universitat Autònoma de Barcelona in 1989. He is currently working toward his PhD degree in the fields of laser surface heat treatment applications, laser beam-shaping, and optical metrology. 


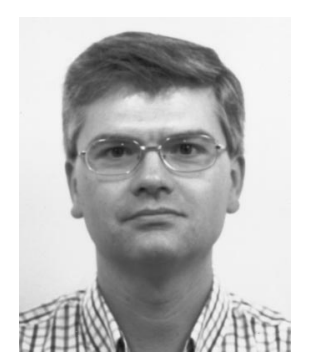

Ferran Laguarta received the MSc degree in physics and the $\mathrm{PhD}$ degree from the Universitat Autònoma de Barcelona in 1981 and 1985 respectively. He was at Stuttgart University in 1985 and 1986 working in the area of infrared and farinfrared lasers. Since September 1985 he has been professor at the Universitat Politècnica de Catalunya (UPC). He is the author of over 50 papers in laser physics, scientific and industrial infrared laser applications, sensing and optical metrology. His current major research interests include laser surface heat-treatment applications, laser beam-shaping techniques and optical surface profilometry. In these fields he is currently the manager of several cooperative research projects financed by private European companies. Since May 1994 he has also been Vice-Rector for Science Policy at UPC. 